\title{
Global stability of the rotating-disc boundary layer with an axial magnetic field
}

\author{
Christian Thomas ${ }^{1}$ and Christopher Davies ${ }^{2} \dagger$ \\ ${ }^{1}$ Department of Mathematics, Imperial College London, London SW7 2AZ, UK \\ ${ }^{2}$ School of Mathematics, Cardiff University, Cardiff CF24 4AG, UK \\ (Received 15 October 2011; revised 14 February 2013; accepted 20 March 2013; \\ first published online 29 April 2013)
}

\begin{abstract}
A numerical study is conducted to investigate the influence of a uniform axial magnetic field on the global linear stability of the rotating-disc boundary layer. Simulation results obtained using a radially homogenized base flow were found to be in excellent agreement with an earlier linear stability analysis, which indicated that an axial magnetic field can locally suppress both convective and absolute instabilities. However, the numerical results obtained for the genuine, radially inhomogeneous, flow indicate that a global form of instability develops for sufficiently large magnetic fields. The qualitative nature of the global instability is similar to that which was observed in a previous study, where mass suction was applied at the rotating disc surface. It is shown that, just as for the case with mass suction, it is possible to explain the promotion of global instability by considering a model that includes detuning effects, which are associated with the radial variation of locally defined absolute temporal frequencies. The recurrence of the same type of instability behaviour when two distinct flow control strategies are implemented, one using suction and the other an axial magnetic field, indicates that the phenomena described by the model may be considered generic.
\end{abstract}

Key words: absolute/convective instability, boundary layer stability, flow control

\section{Introduction}

The behaviours that are found when a magnetic field is imposed upon an electrically conducting fluid flow can have important consequences for a variety of engineering applications. They also merit attention because there can be an exhibition of interesting changes from the behaviour that is usually obtained for non-conducting flow problems. Electromagnetic stirring and casting of liquid metals and the stimulation of the growth of crystals (Organ \& Riley 1987; Hicks \& Riley 1989) are examples of industrial processes where magnetic fields are utilized. Magnetohydrodynamical effects can also influence the stability characteristics of fluid systems, by suppressing or promoting flow instability and transition from a laminar to a turbulent state. (For an example of a recent investigation, that demonstrates the stabilization of an oscillatory laminar flow, see Thomas, Bassom \& Davies (2010).) 
Several studies have indicated that magnetic fields can be used to control the onset of instability for the rotating-disc boundary layer that is generated when a radially unbounded solid disc rotates beneath an initially stationary, incompressible, viscous body of electrically conducting fluid. Circular magnetic fields applied to the rotating flow have a tendency to increase the boundary-layer thickness and destabilize disturbances from the basic state (Pao 1968; Kumar, Thacker \& Watson 1987), while axial magnetic fields directed normal to the disc surface are known to have a stabilizing effect (Sparrow \& Cess 1962; Suryaprakasarao \& Gupta 1966; Thacker, Watson \& Kumar 1990). For the latter case, which will be investigated further in the present study, the changes in the character of the base flow that are due to the magnetic field are qualitatively similar to those which are found when uniform mass suction is applied at the disc surface. In particular, the magnitude of the radial component of the base flow decreases and the azimuthal component (in the nonrotating frame of reference) decays more rapidly away from the disc. It is these kinds of alteration to the base flow that lead to the local stabilization of small-amplitude disturbances.

For non-conducting fluids placed above a rotating disc, the boundary-layer flow has a long and rich history of both theoretical and experimental investigation (Saric, Reed $\&$ White 2003). The profile of the basic flow, which can be obtained using a similarity solution to the Navier-Stokes equations due to von Kármán (1921), is qualitatively similar to that of the boundary layer that forms over a swept wing. Both flows have inflection points and are susceptible to the cross-flow instability that was discovered by Gray (1952) and Gregory, Stuart \& Walker (1955). An inferred kinship between the instability mechanisms has often been taken as the prime motive to study rotating-disc boundary layers, the real aim being to cast some light on swept-wing behaviour. Nevertheless, there are at least two further modes of instability that can occur for the rotating-disc boundary layer, which were identified by Faller \& Kaylor (1966) and Mack (1985). The cylindrical geometry of the rotating-disc flow also distinguishes it from swept-wing flows: the periodicity of the azimuthal co-ordinate can be interpreted as creating, along the tangential direction in planes parallel to the disc, an enclosed space for the propagation of disturbances, which has no immediate counterpart in the swept-wing geometry.

For both non-conducting and conducting fluids, with or without an axial magnetic field, the linear stability characteristics of the rotating-disc boundary layer can be determined, at least locally near a specified non-dimensional radius, using a sixth-order linear system of equations. This system defines a local dispersion relationship

$$
D(\alpha, \omega ; \operatorname{Re}, \beta, m)=0,
$$

where $\alpha$ and $\beta$ are the respective radial and azimuthal wavenumbers for the presumed normal-mode form of the disturbances, $\omega$ is the corresponding temporal frequency and $R e$ is the Reynolds number based on the constant boundary-layer thickness and the local circumferential speed of the disc rotation. The parameter $m$ gives a nondimensional measure of the axial magnetic field strength. It is important to note that the use of a base-flow homogeneity approximation along the radial direction is an essential step in the construction of the dispersion relation (1.1). The Reynolds number $R e$ is inherently local because it is equal to the non-dimensional radius at the radial location where the base flow is homogenized. (A few further comments are given shortly in connection with this. For more details and a careful discussion, see, for example, Davies, Thomas \& Carpenter (2007).) 
For the non-conducting flow case, for which $m=0$, Lingwood $(1995,1997)$ showed that absolute instability appears for Reynolds numbers greater than 507.3, which was noted to be very near to the typically observed locations for transition to turbulence in physical experiments. Jasmine \& Gajjar (2005) extended the analysis of Lingwood to non-zero $m$ and found that the critical Reynolds numbers for convective and absolute instabilities were both raised significantly with the introduction of a uniform axial magnetic field. For the conducting flow with $m=0.5$, the critical Reynolds number for absolute instability was given as $R e=1091.9$, more than double that found for the non-conducting flow.

It should be reiterated that the linear stability investigations of Lingwood (1995), Lingwood (1997) and Jasmine \& Gajjar (2005) were all carried out using the homogenized flow approximation that facilitates the construction of a local dispersion relation. In order to obtain a separable eigensystem, which involves only ordinary differential equations, the variation of the base flow along the radial direction is artificially frozen, which permits a normal-mode treatment for the radial dependence of the disturbances. This simplification of the basic flow state, although convenient for the purposes of the stability analysis, leaves out a characteristic physical feature of rotating-disc boundary-layer flows. Namely, the attribute that the radial and azimuthal velocity components of the basic state are linearly proportional to the radius.

In the current investigation we will conduct a study of the linear stability of the genuine, radially dependent, rotating-disc boundary layer with an imposed axial magnetic field, i.e. without making use of any base-flow homogenization. This is achieved by means of direct numerical simulations for impulsively excited linear disturbances, which were undertaken using the numerical methods and velocity-vorticity formulation that were described by Davies \& Carpenter (2001). A similar simulation scheme had previously been deployed to study the evolution of disturbances in the non-conducting rotating-disc boundary layer (Davies \& Carpenter 2003; Davies et al. 2007) and extended to cases where there is uniform mass transfer applied at the disc surface (Thomas \& Davies 2010). The changes that were needed to incorporate the magnetic force term will be described further on in the paper.

In the earlier numerical simulation studies for the flow with no mass transfer, the results indicated that the long-term behaviour of impulsively excited disturbances was convective; perturbations were found to continually propagate radially outwards, even when they originated within or passed into absolutely unstable regions of the flow. Thus, the influence of the radial inhomogeneity of the basic state was considered to be globally stabilizing. Within the confines of the linear approximation that was used, the absolute instability predicted from the local dispersion relation only made its presence felt through a form of transient temporal growth; at any given radial location, the disturbance would eventually begin to decay. The occurrence of this form of behaviour was subsequently confirmed by observations that were made in a physical experiment conducted by Othman \& Corke (2006), for disturbances that were carefully excited so as to have a sufficiently small initial amplitude.

When the numerical simulations were extended to introduce mass injection at the disc surface, the flow remained globally stable, even though it became locally more unstable. However, when mass suction was applied instead of injection, the effects on the global and local stability were reversed. For sufficiently large suction it was found that disturbances became globally unstable, despite the fact that they were locally stabilized. Some more details about the novel nature of this instability will be given later, when we describe how similar behaviour arises in the presence of an axial magnetic field. 
An explanation of the global stability behaviour was first given in Davies et al. (2007) for the case with no mass transfer, and then generalized to encompass the whole class of rotating disc boundary flows with mass transfer in Thomas \& Davies (2010). In both of these investigations, successful comparisons were made between numerical simulation results and impulse solutions of the linearized complex Ginzburg-Landau equation. The relevant impulse solutions were originally found by Hunt \& Crighton (1991).

Healey (2010) made a speculative extension of the modelling work of Davies et al. (2007) and Thomas \& Davies (2010), in which the effects of a finite radial extent for the rotating disc were accounted for by the ad hoc imposition of a boundary condition on the impulse solutions of the Ginzburg-Landau equation. Subsequent physical experiments, which were carefully conducted by Imayama, Alfredsson \& Lingwood (2013), have failed to provide any firm evidence to support Healey's contention that for finite discs there would a global instability with an onset that varied according to the disc radius.

The elucidation of the behaviour identified in the previously conducted numerical simulations hinges on the fundamental notion of temporal frequency detuning. If a disturbance propagates within regions of a flow where the local absolute temporal frequency varies in space, then dependent upon a competition between changes in the frequency and corresponding changes in the absolute temporal growth rates, either global stability or global instability can ensue. The precise balance that determines the global stability is also mediated by dispersion and diffusion effects.

A primary motivation for studying the rotating-disc boundary-layer flow with an axial magnetic field, aside from its intrinsic interest as a distinct physical problem, is to explore whether or not the kind of global stability behaviour that was identified for the family of flows with mass transfer can be considered generic. More specifically, it may be asked: Can any variations that are discovered between globally stable and globally unstable behaviour be accounted for, once more, by considering a model that includes detuning effects? We shall see that this question can in fact be answered affirmatively.

The remainder of the paper is organized as follows. The subsequent section briefly describes the basic flow equations and the amendments to the velocity-vorticity formulation of Davies \& Carpenter (2001) that were needed in order to incorporate the effects of the axial magnetic field when tracking the evolution of disturbances. In $\S 3$, numerical simulation results are presented. The main focus will be on the development of disturbances in the inhomogeneous flow for the specific choice of the non-dimensional axial magnetic field parameter $m=0.5$. This particular case was selected because it exemplifies the globally unstable behaviour that is of central interest here. We also consider, more briefly, simulation results that display how the qualitative nature of the behaviour varies when $m$ is altered. In the following $\S 4$ an explanatory account is given for this variation in the behaviour. A few final remarks regarding our findings are presented in $\S 5$.

\section{Formulation}

Consider a disc of indefinitely large radial extent, rotating with a constant angular velocity $\Lambda^{*}$ about a vertical axis that passes through the centre of the disc. The rotating-disc rests beneath a semi-infinite stationary body of incompressible, electrically conducting, viscous fluid. Cylindrical polar co-ordinates are used to define our system, where $r^{*}, \theta$ and $z^{*}$ refer to the respective radial, azimuthal and axial 
directions. The frame of reference is taken to be at rest with respect to disc. A uniform magnetic field $\boldsymbol{B}^{*}=B^{*} \boldsymbol{k}$ is imposed, where $\boldsymbol{k}$ is the unit vector parallel to the axial direction and $B^{*}$ is the magnetic field strength. (Here and immediately below, an asterisk denotes a dimensional quantity.)

The impact of the magnetic field on the fluid motion is represented by a Lorentz force, $\boldsymbol{L}^{*}=\boldsymbol{J}^{*} \wedge \boldsymbol{B}^{*}$, where $\boldsymbol{J}^{*}=\sigma\left(\boldsymbol{E}^{*}+\boldsymbol{U}^{*} \wedge \boldsymbol{B}^{*}\right)$ is the current density, for an electric field $\boldsymbol{E}^{*}$, velocity $\boldsymbol{U}^{*}=\left\{U_{r}^{*}, U_{\theta}^{*}, U_{z}^{*}\right\}$ and electrical conductivity $\sigma$. For the sake of simplicity, we suppose that the direct effect of the electric field is negligible in determining the Lorentz force and that the magnetic field is not altered by the fluid motion. These assumptions can be applied when the magnetic Reynolds number $R e_{m}$ is found to be such that $R e_{m} / R e=\mu_{0} \sigma v \ll 1$, where $\mu_{0}$ is the magnetic permeability constant and $v$ the kinematic viscosity. For the particular case of liquid Mercury at room temperature, it is easily checked that the physical values taken by $\mu_{0}, \sigma$ and $v$ do in fact ensure that the Reynolds number for the fluid motion is several orders of magnitude greater than the associated magnetic Reynolds number.

Making use of the simplifying assumptions that were just stated, we obtain the expression

$$
\boldsymbol{L}^{*}=\sigma B^{* 2}\left(\boldsymbol{U}^{*} \wedge \boldsymbol{k}\right) \wedge \boldsymbol{k},
$$

for the magnetic force term that appears in the momentum equations for the fluid motion. It may be noted that this term has a quadratic dependence on the strength of the magnetic field and thus remains unaltered if the direction of the magnetic field is reversed.

\subsection{Basic state}

A similarity type of solution may be determined by introducing von Kármán (1921) similarity variables such that

$$
\left\{U_{r}^{*}, U_{\theta}^{*}, U_{z}^{*}\right\}=\left\{r^{*} \Lambda^{*} F(z), r^{*} \Lambda^{*} G(z), \delta^{*} \Lambda H(z)\right\},
$$

where $F, G$ and $H$ represent the respective non-dimensional radial, azimuthal and axial basic flow profiles. The non-dimensional co-ordinate that is used along the wall normal direction is $z=z^{*} / \delta^{*}$, where $\delta^{*}=\left(v / \Lambda^{*}\right)^{1 / 2}$ is the constant boundarylayer thickness. On substituting (2.2) into the Navier-Stokes equations for the given cylindrical configuration, the following system of ordinary differential equations is obtained

$$
\begin{gathered}
F^{2}+F^{\prime} H-(G+1)^{2}+m F=F^{\prime \prime}, \\
2 F(G+1)+G^{\prime} H+m(G+1)=G^{\prime \prime}, \\
2 F+H^{\prime}=0,
\end{gathered}
$$

where a prime denotes differentiation with respect to $z$ and $m=\sigma B^{* 2} /\left(\rho \Lambda^{*}\right) \geqslant 0$ is the non-dimensional magnetic field parameter, with $\rho$ being the fluid density. Note that the invariance of the magnetic force term with respect to reversal of the magnetic field direction is implicit in the restriction that $m$ cannot be negative.

Equations $(2.3 a)-(2.3 c)$ need to be solved subject to the boundary conditions

$$
\begin{gathered}
F(0)=G(0)=H(0)=0, \\
F(z \rightarrow \infty)=0, \quad G(z \rightarrow \infty)=-1 .
\end{gathered}
$$

Numerical solutions for the profile functions $F, G$ and $H$ were determined and found to be in good agreement whose those that have been obtained by previous investigators 
(Thomas 2007). As was noted in the introduction, two of the most salient features of these solutions are that, as the magnetic field is increased, the magnitude of the radial velocity component $F$ diminishes and the azimuthal velocity component $G$ approaches its far-field value at infinity more rapidly. The latter behaviour can be interpreted as being a kind of boundary layer thinning, but it should be remarked that this takes place with respect to a wall-normal co-ordinate that has already been scaled using the constant dimensional boundary-layer thickness $\delta^{*}$.

The spatial variation of the basic flow may be represented in non-dimensional form as

$$
\boldsymbol{U}_{B}=\left(\frac{r}{\operatorname{Re}} F(z), \frac{r}{\operatorname{Re}} G(z), \frac{1}{\operatorname{Re}} H(z)\right),
$$

where a local Reynolds number $R e$ is defined as

$$
R e=r_{a}^{*}\left(\frac{\Lambda^{*} \delta^{*}}{v}\right)=\frac{r_{a}^{*}}{\delta^{*}}=r_{a},
$$

for some reference radial position $r_{a}^{*}$. As has been mentioned before, previous theoretical studies concerning the stability of rotating-disc boundary layers, such as those undertaken by Lingwood (1995, 1997) and Jasmine \& Gajjar (2005), have enforced a radial homogeneity approximation; the radial dependence of the basic flow (2.4) is removed, by the simple expedient of setting $r=r_{a}$, whenever the base flow appears as a multiplier in the governing equations that determine the development of linear disturbances.

\subsection{Disturbance equations}

Perturbations to the basic flow (2.4) are considered using the decomposition

$$
\boldsymbol{U}=\boldsymbol{U}_{B}+\boldsymbol{u}, \quad \boldsymbol{\Omega}=\boldsymbol{\Omega}_{B}+\boldsymbol{\omega},
$$

where the undisturbed vorticity is $\Omega_{B}=\nabla \wedge \boldsymbol{U}_{B}$ and the perturbation variables are given as

$$
\boldsymbol{u}=\left\{u_{r}, u_{\theta}, u_{z}\right\}, \quad \boldsymbol{\omega}=\left\{\omega_{r}, \omega_{\theta}, \omega_{z}\right\} .
$$

Selecting the perturbation components $\left\{\omega_{r}, \omega_{\theta}, u_{z}\right\}$ as the primary variables, the linearized disturbance evolution can be determined using the following set of governing equations, obtained by a straightforward amendment of those that were derived in Davies \& Carpenter (2001):

$$
\begin{aligned}
& \frac{\partial \omega_{r}}{\partial t}+\frac{1}{r} \frac{\partial N_{z}}{\partial \theta}-\frac{\partial N_{\theta}}{\partial z}-\frac{2}{R e}\left(\omega_{\theta}+\frac{\partial u_{z}}{\partial r}-\frac{m}{2}\left(\omega_{r}-\frac{1}{r} \frac{\partial u_{z}}{\partial \theta}\right)\right) \\
&=\frac{1}{R e}\left(\left(\nabla^{2}-\frac{1}{r^{2}}\right) \omega_{r}-\frac{2}{r^{2}} \frac{\partial \omega_{\theta}}{\partial \theta}\right), \\
& \frac{\partial \omega_{\theta}}{\partial t}+\frac{\partial N_{r}}{\partial z}-\frac{\partial N_{z}}{\partial r}+\frac{2}{R e}\left(\omega_{r}-\frac{1}{r} \frac{\partial u_{z}}{\partial \theta}+\frac{m}{2}\left(\omega_{\theta}+\frac{\partial u_{z}}{\partial r}\right)\right) \\
&=\frac{1}{R e}\left(\left(\nabla^{2}-\frac{1}{r^{2}}\right) \omega_{\theta}+\frac{2}{r^{2}} \frac{\partial \omega_{r}}{\partial \theta}\right), \\
& \nabla^{2} u_{z}=\frac{1}{r}\left(\frac{\partial \omega_{r}}{\partial \theta}-\frac{\partial\left(r \omega_{\theta}\right)}{\partial r}\right)
\end{aligned}
$$


where

$$
\nabla^{2} f=\frac{\partial^{2} f}{\partial r^{2}}+\frac{1}{r} \frac{\partial f}{\partial r}+\frac{1}{r^{2}} \frac{\partial^{2} f}{\partial \theta^{2}}+\frac{\partial^{2} f}{\partial z^{2}},
$$

and

$$
\boldsymbol{N}=\left\{N_{r}, N_{\theta}, N_{z}\right\}=\boldsymbol{\Omega}_{B} \times \boldsymbol{u}+\boldsymbol{\omega} \times \boldsymbol{U}_{B} .
$$

The linearized convective term $N$ depends upon the remaining perturbation components $\left\{u_{r}, u_{\theta}, \omega_{z}\right\}$, but these so-called secondary variables may be eliminated since they can be defined explicitly in terms of the primary variables. (Further details are provided in Davies \& Carpenter (2001)).

It may be seen that, due to the imposed magnetic field, the vorticity transport equations are modified by the inclusion of additional terms that are multiplied by the magnetic field parameter $m$. These represent the non-dimensional version of the curl of the magnetic force that is given in (2.1). In each of the radial and azimuthal vorticity transport $(2.8 a, b)$ the extra terms are proportional, respectively, to the wall-normal derivative of the azimuthal and the radial component of the perturbation velocity; the definition of the vorticity has been deployed to conveniently rewrite these secondary variable derivatives using the primary variables only.

Since we are only concerned with linear disturbances, it is possible to consider the independent development of modes of the general form

$$
\{\boldsymbol{u}, \boldsymbol{\omega}\}=\{\hat{\boldsymbol{u}}, \hat{\boldsymbol{\omega}}\} \mathrm{e}^{\mathrm{i} n \theta},
$$

where $n$ is the azimuthal mode number. The cylindrical geometry of the flow configuration means that $n$ is restricted to take integer values. For all of the simulations that we shall report, a disturbance with a chosen value of $n$ was impulsively excited by prescribing a deformation in the height of the disc surface. This was fed into the boundary conditions on the disturbance using a linearization. The forcing was taken to have a high degree of radial localization, as well as being applied for only a very short time duration, which ensured that a broad spectrum of temporal frequencies was seeded. Details about the specific form of the impulse that was used can be found in Thomas \& Davies (2010).

\section{Results}

Numerical simulations were conducted for a range of magnetic field parameter values up to $m=0.5$. Particular values of $m$ were selected so as to check that the simulation results were in good agreement with the published data given by Jasmine \& Gajjar (2005) for the local stability behaviour. This provided a careful validation of the computer code, which had been amended to incorporate the effects of the magnetic field. In order to facilitate comparisons, we had to artificially homogenize the basic flow, suppressing its radial variation. Full details of the excellent matching that was obtained are documented in Thomas (2007).

For disturbances developing in the genuine, radially inhomogeneous, base flow, we will indicate the typical character of the numerical simulation results by describing the behaviour for $m=0.5$. Following on from our reports for this particular case, the qualitative variation of the behaviour with changes in $m$ will be considered.

\subsection{Illustration for the case $m=0.5$}

We first examine the evolution of a disturbance with an azimuthal mode number $n=119$ that is impulsively excited at the radial location $r_{e}=1092$. This position 

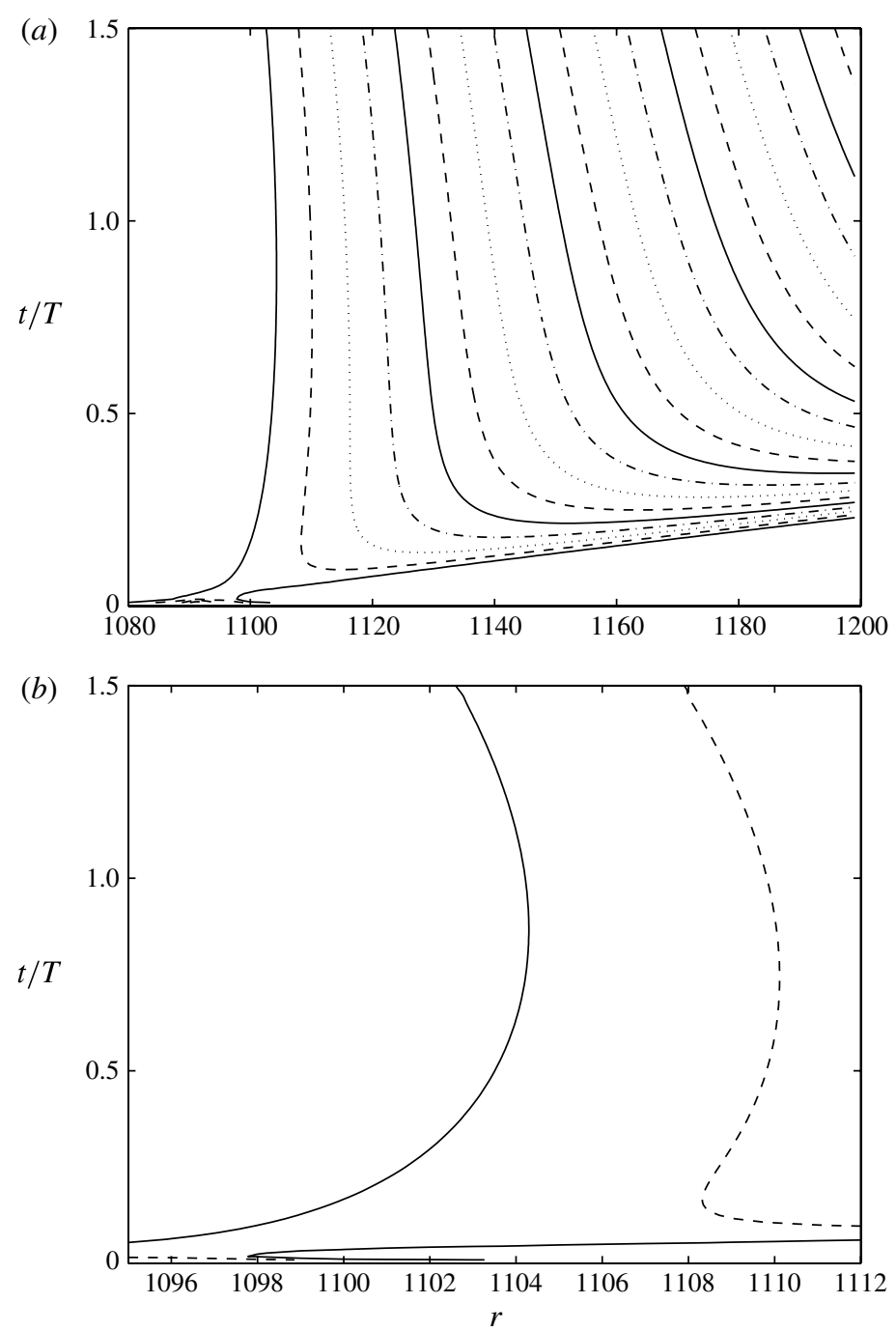

FIGURE 1. Spatial-temporal development of azimuthal vorticity at the disc surface $\left|\omega_{\theta}\right|_{z=0}$ for an impulsively excited disturbance in the flow with $m=0.5$ : $(a)$ displayed with a larger radial range; $(b)$ for a reduced radial range. The azimuthal mode number is $n=119$ and the disturbance was excited at $r_{e}=1092$. Contours are plotted using a logarithmic scaling. The amplitude changes by a factor of two between successive contours.

is selected so as to be very near to that for which there would be critical absolute instability, according to the results of the stability analysis for the homogenized version of the flow. The spatial-temporal disturbance development for the inhomogeneous flow is displayed in figure 1(a) over the radial range $1080 \leqslant r \leqslant 1200$. The time is measured using a globally valid scaling, to remove the implicit local dependence that would otherwise appear, because the time period for the disc rotation is given by $T=2 \pi R e$ when the usual form of non-dimensionalization is employed. (The usage of a global time scale also shows up later, in factors of $R e$ that are included when we plot temporal frequencies and growth rates.) The wavepacket 
development is plotted using contours of the amplitude of the azimuthal perturbation vorticity $\omega_{\theta}$ at the wall. The leading edge of the disturbance can be seen to propagate to the right of the original impulse in figure $1(a)$ and is clearly travelling with a non-zero velocity. By contrast, the trailing edge is almost parallel to the time axis and moves with what might seem to be a diminishing velocity. However, if we reduce the range of the radii over which the trailing edge is examined, as in figure $1(b)$, then it may be seen that at about the time $t / T \approx 0.7$, the edge reverses in direction and the disturbance begins to propagate radially inwards, with a small but increasing velocity. Hence, the two edges of the disturbance wavepacket eventually propagate in opposite directions, suggesting that a global form of instability is developing. The long term character of the disturbance can thus be seen to be markedly different from that identified by Davies \& Carpenter (2003) for the non-conducting flow. Instead, the behaviour is qualitatively similar to that which was observed by Thomas \& Davies (2010) when they considered the rotating-disc boundary-layer modified by uniform suction, applied with sufficient strength.

Some more insight into the disturbance behaviour can be obtained by using the simulation data to compute the following complex-valued logarithmic derivative

$$
\omega=\frac{\mathrm{i}}{A} \frac{\partial A}{\partial t},
$$

where $A$ is a chosen measure of the disturbance amplitude. As before, the perturbation azimuthal vorticity at the disc surface is selected to give the amplitude. For any given radial position, the real and imaginary parts of $\omega$ may be interpreted as being the instantaneously defined temporal frequency and growth rate, respectively.

Figure 2 displays temporal frequencies and growth rates obtained from the same inhomogeneous flow simulation that was considered immediately above. The results are plotted for four successive radial positions, including the centre of the impulse. Additional solid-dotted lines, denoted $\mathrm{P}$, give frequencies and growth rates that were found from a corresponding homogenized flow simulation with $R e=1092$. For the homogeneous case, results are only shown for the location where the impulse was applied. It may be seen that the frequency is constant for the time duration considered and is given approximately as $\omega_{r} R e \approx-33$; because of the imposed radial homogeneity, essentially the same behaviour was found at other nearby radial locations. It may also be observed that the growth rate for the homogenized flow asymptotes towards zero, which is consistent with our having chosen the parameters to coincide with critical absolute instability.

The temporal frequencies from the inhomogeneous flow simulation display features that match those of the earlier study of Davies \& Carpenter (2003), for the rotatingdisc boundary layer without a magnetic field. Just as was found for the $m=0$ case, the temporal frequencies vary in a systematic fashion with the radial position, but all show an eventual decrease in magnitude as the time increases and fail to approach any constant value.

The behaviour of the corresponding temporal growth rates does not, however, agree with that which was observed for the non-conducting flow. The temporal growth rates develop in a distinctive manner that looks similar to that which was identified for disturbances in the rotating disc with uniform mass suction (Thomas \& Davies 2010). In figure $2(b)$, the growth rates are plotted over the full interval of times from the simulation, while in figure $2(c)$, the time axis has been magnified and the plots are given for a much reduced range of later times. For each of the radial positions considered, the growth rates are found to show an eventual increase. After 

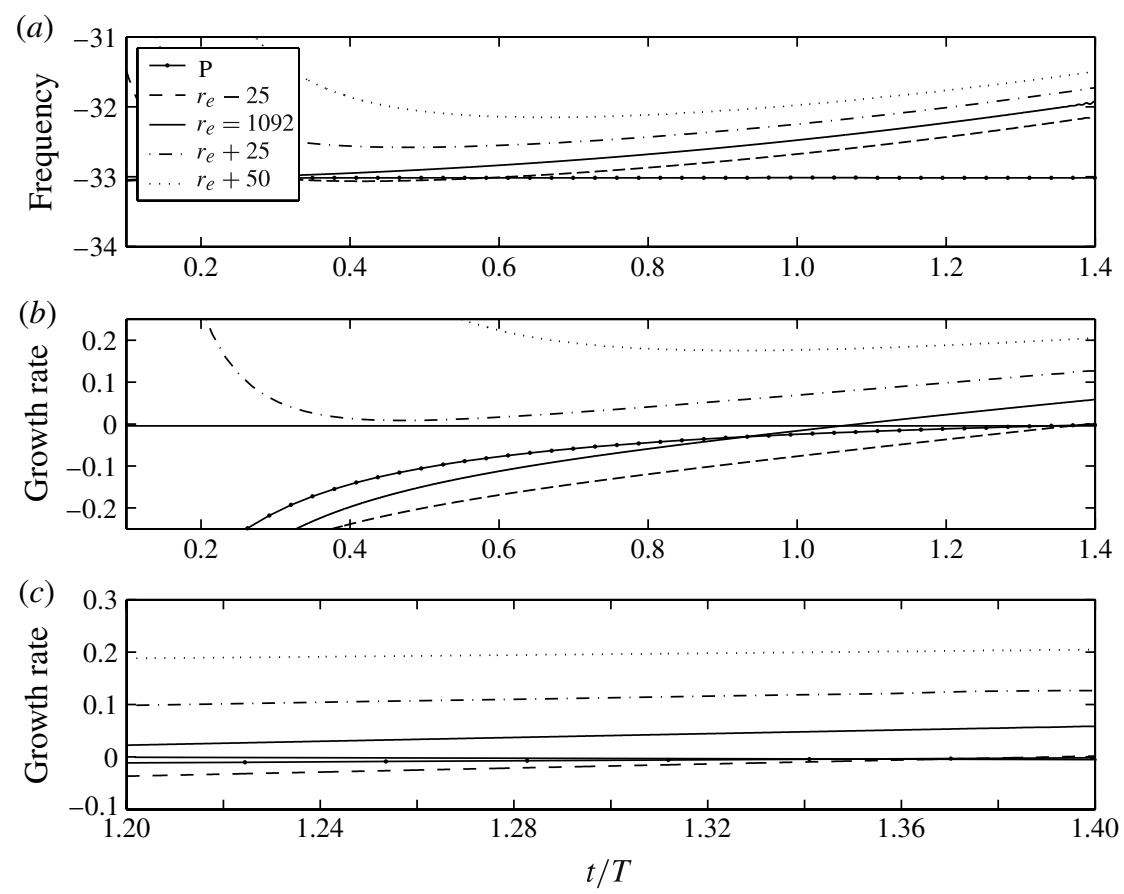

FIGURE 2. Instantaneously defined (a) temporal frequencies $\omega_{r} R e$ and $(b, c)$ temporal growth rates $\omega_{i} R e$ for the same disturbance excited at $r_{e}=1092$ as was shown in figure 1 . The evolution is plotted for four different radial positions, $r_{e}-25, r_{e}, r_{e}+25$ and $r_{e}+50$. The solid-dotted lines labelled with a $\mathrm{P}$ are for the corresponding development in the homogenized version of the flow with $R e=1092$. The temporal growth rates are displayed over the full time interval in $(b)$ and for a reduced range of times towards the end of this interval in $(c)$.

sufficient time, positive growth is experienced at all of the selected radial locations, including the position $r_{e}-25$ that is located radially inwards from the origin of the excitation. Unfortunately, the simulations could not be continued indefinitely, to determine the disturbance behaviour for an arbitrarily large period of time; given enough time, artificial effects contaminate the results because the maxima of the disturbance wavepacket grows enormously, which leads to convergence problems and also makes it difficult to control reflections from the computational boundaries. Thus, we cannot completely dispel the possibility that the growth rates will at some later time begin to decrease and perhaps change sign, so as to indicate temporal decay. Nevertheless, our simulation results strongly suggest that the rotating-disc flow with a magnetic field parameter $m=0.5$ is in fact globally unstable. It must be emphasized that the behaviour that characterizes this instability differs markedly from what is commonly found in other globally unstable flows, such as bluff body wakes, in that there is no selection of any dominant global temporal frequency as the disturbance develops. The absence of a global frequency was also a distinguishing feature of the instability that was discovered when uniform suction was deployed as the means of modifying the basic flow (Thomas \& Davies 2010).

We now continue our investigation for the case $m=0.5$, by considering simulation results for the evolution of a perturbation with the same azimuthal mode number as before, but which is now excited at a large distance radially inboard from the region 


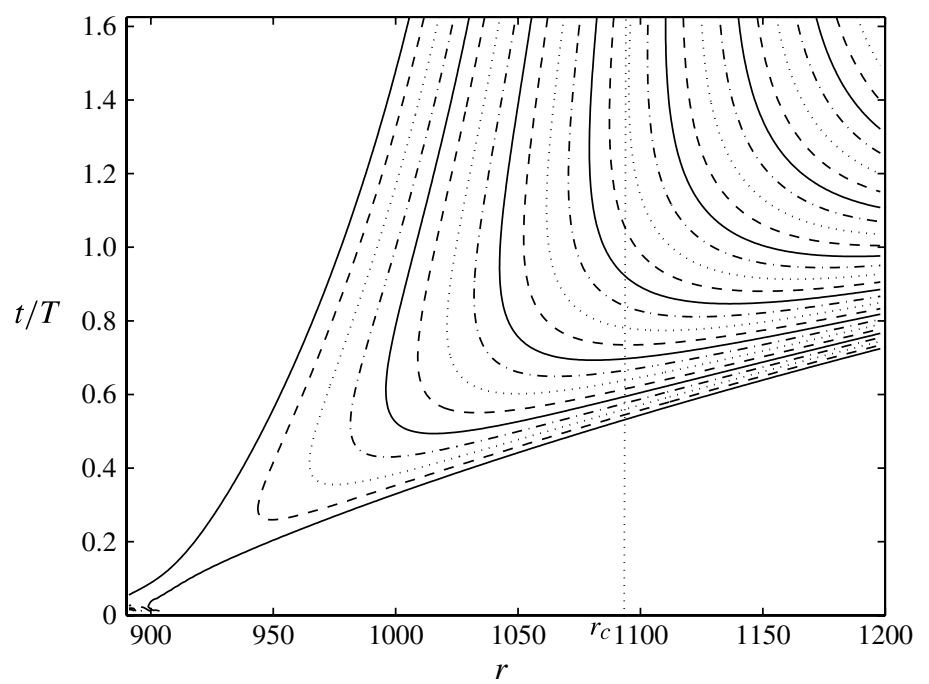

FIGURE 3. Spatial-temporal development of $\left|\omega_{\theta}\right|_{z=0}$ for an impulsively excited disturbance in the flow with $m=0.5$. The azimuthal mode number is again $n=119$, but the disturbance is now excited at $r_{e}=892$. Contours are separated by factors of two.

where the homogenized flow analysis predicts that there will be absolute instability. Letting $r_{c}=1092$ denote the location for which absolute instability is expected to set in, the impulse is centred at $r_{e}=r_{c}-r_{s}=892$, where we have set $r_{s}=200$ to be the radially inward shift into the region where only convectively unstable behaviour is anticipated. Our intention is to highlight some contrasts with the disturbance evolution that was found in a similar simulation, with the same choice of $r_{s}$, that was reported by Davies \& Carpenter (2003) for the non-conducting flow. When they examined the disturbance wavepacket they found that, as might be expected, it passed through the region of convective instability and into the region of absolute instability. More interestingly, the simulation results indicated that the trailing edge would continue to travel radially outwards, with an increasingly positive velocity, even as it approached and subsequently moved beyond the radial position $r_{c}$ where the onset of absolute instability had been predicted.

For our present study with $m=0.5$, figure 3 depicts the spatial-temporal development of a disturbance excited at the chosen radial location $r_{e}=892$. A vertical dotted line is used to mark the critical radius $r_{c}$. It may be seen that as the wavepacket develops, the trailing edge of the disturbance propagates radially outwards. However, the velocity associated with the trailing edge does not appear to increase as it approaches $r_{c}$, as it did in the corresponding simulation for $m=0$. Instead, the edge seems to move with a diminishing velocity. From a consideration of the contour lines drawn within the wavepacket, it might be inferred that the trailing edge will eventually slow down to a zero velocity near to $r_{c}$. Unfortunately, no categorical assertion can be made about this behaviour, in view of the finiteness of the time interval over which the simulation could be successfully continued. Likewise, there is no unequivocal evidence to confirm that the trailing edge will, given enough time, reverse its direction of travel, in the same fashion as was described previously for the case where the disturbance was excited at $r_{c}$. 

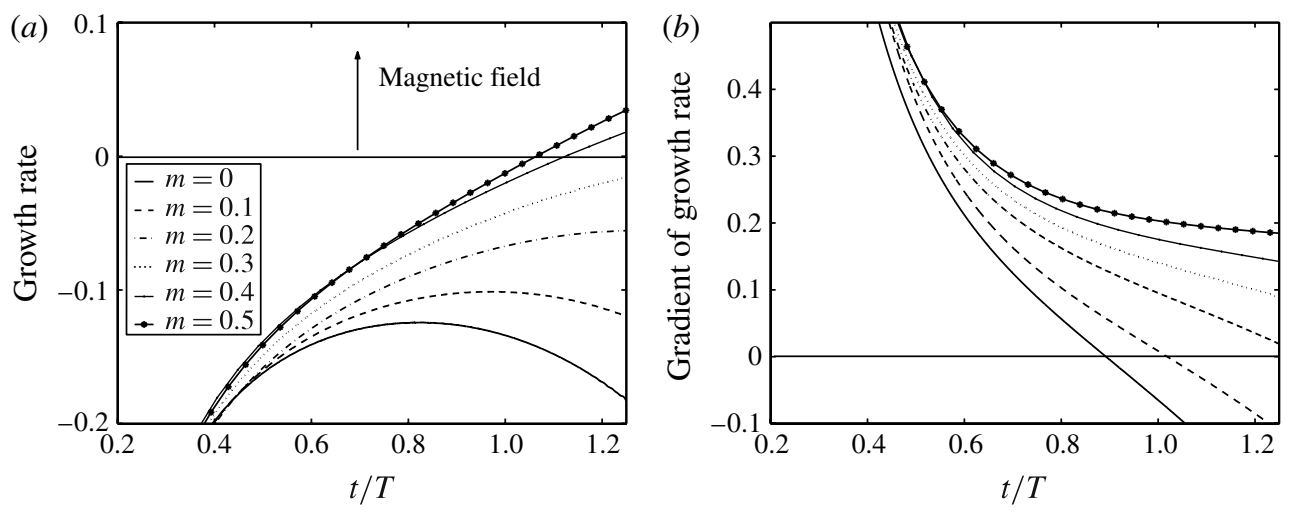

FIGURE 4. Instantaneous growth rates and their gradients for disturbances at the critical point of absolute instability for flows with various values of the magnetic parameter. Solid line: $m=0,\left(r_{e}=508, n=68\right)$; dashed line: $m=0.1,\left(r_{e}=595, n=76\right)$; dashed-dotted line: $m=0.2,\left(r_{e}=696, n=86\right)$; dotted line: $m=0.3,\left(r_{e}=813, n=96\right)$; solid-dotted line: $m=0.4,\left(r_{e}=946, n=107\right)$; solid-star line: $m=0.5\left(r_{e}=1092, n=113\right)$.

\subsection{Variation of behaviour with $m$}

Having used the case $m=0.5$ to illustrate the kind of globally unstable behaviour that can occur when the base flow is modified by the presence of an axial magnetic field, we now turn to consider how the behaviour varies when the strength of the magnetic field is altered. Figure 4 displays the evolution of the temporal growth rates, together with their gradients, for disturbances that are all excited at radial locations $r_{e}=r_{c}$ that correspond to critical absolute instability. Results are given for six different flows, with magnetic field parameters taken at equally spaced increments in the range between $m=0$ and $m=0.5$. The data for each of the plotted lines are as given in the caption; all of them are taken at the centre of the impulse. Initially, the growth rates for every case show the same trend of increase from negative values within the lower half of the plane. However, after approximately $t / T=0.5$ there are significant differences amongst the behaviours exhibited by the growth rates. For relatively small magnetic fields, $m<0.2$, the growth rates reach a maximum before reversing downwards and decreasing. Temporal decay is observed over the whole of the time interval for these cases. By contrast, the growth rates for the larger $m$ values continue to increase throughout the finite time period that is considered. For the flows with $m=0.2,0.3$, it would appear, from an inspection of the matching gradients shown in the bottom half of the figure, that the trend is such that the growth rates may eventually decrease to become negative. As with the smaller values of $m$, this would mean that the disturbance has temporal decay attributes which indicate that there is global stability. The long-term behaviour seems a little clearer for the flows with magnetic field parameters $m=0.4,0.5$. The growth rates continue to increase and become positive for the latter portion of the time interval. It also appears that the corresponding growth rate gradients, although themselves decreasing, may be bounded below by a positive constant, which would signify a disturbance with an increasingly large degree of amplification.

Although it was feasible to conduct numerical simulations for the rotating-disc boundary layer with magnetic field strengths greater than those that have just been considered, such simulations were not undertaken in any systematic manner. This 
was simply because the critical parameters for the absolute instability, as determined using a local stability analysis based on the homogeneous flow approximation, are not currently available in the literature for $m>0.5$; so the selection of appropriate parameters for the simulations would be hampered. Some further investigation would be preferable, to confirm that larger axial magnetic fields do in fact continue to promote globally unstable behaviour, when inhomogeneous flow effects are taken into consideration. Fortunately, for our purposes, it was possible to identify a changeover from global stability to global instability within the limited range of $m$ values that we chose to study.

\section{Comparison with model impulse solutions}

In their study of the global stability of the rotating-disc boundary layer with mass transfer at the disc surface, Thomas \& Davies (2010) were able to offer an explanation of their simulation results by making careful comparisons with behaviour displayed by impulse solutions of the linearized Ginzburg-Landau equation. Similar comparisons can be made for the present case. Rather than repeating the same kind of detailed analysis as was presented in the earlier study, we will confine ourselves to showing how it is possible to capture the essential features of the disturbance evolution that are conveyed in the growth rate plots given in figure 4.

The linearized Ginzburg-Landau equation may be written in the form:

$$
\frac{\partial A}{\partial t}+U \frac{\partial A}{\partial r}=\mu A+\gamma \frac{\partial^{2} A}{\partial r^{2}}
$$

where $A(r, t)$ is some measure of the disturbance amplitude at the spatial location $r$ and time $t$. The terms multiplied by the quantities $\mu, U$ and $\gamma($ where $\operatorname{Re}(\gamma)>0$ ) are included to specify, respectively, the behaviour due to temporal growth/oscillation, flow convection and diffusion/dispersion. All three of these quantities need to be taken as constants for a spatially homogeneous flow.

If we allow $\mu$ to vary linearly by setting $\mu=\mu_{0}+\mu_{1} r$, while still keeping both $U$ and $\gamma$ constant, then we obtain a simple model that may be used to capture the effects of local alterations in the stability properties when the flow is inhomogeneous. The real part of $\mu_{1}$ provides a measure for incorporating spatial variation in the temporal growth that disturbances will exhibit, while the imaginary part encompasses the corresponding dependency in the temporal oscillation frequency. Alterations in the disturbance frequency, when the spatial position is changed, can easily be overlooked because attention is usually focused on changes in the growth rate. We designate as detuning the phenomena where the temporal frequency predicted for an instability depends to a significant extent on the particular spatial location that is being considered.

When a linear variation is prescribed for $\mu$, it is possible to derive an impulse solution of the Ginzburg-Landau equation that has the following analytic form (Hunt \& Crighton 1991; Thomas \& Davies 2010):

$$
G(r, t)=\sqrt{\frac{1}{4 \pi \gamma t}} \exp \left(-\frac{r^{2}}{4 \gamma t}+\frac{1}{2} \mu_{1} r t+\frac{1}{12} \mu_{1}^{2} \gamma t^{3}\right) \exp \left(\mathrm{i}\left(\alpha_{0} r-\omega_{0} t\right)\right),
$$

where

$$
\omega_{0}=\mathrm{i}\left(\mu_{0}-\frac{U^{2}}{4 \gamma}\right), \quad \alpha_{0}=-\mathrm{i} \frac{U}{2 \gamma}
$$


For the sake of convenience, the spatial co-ordinate $r$ is shifted so that $r=0$ defines the centre of the impulse. The explicit dependency of the solution on the convection velocity $U$ has been suppressed by introducing the temporal frequency $\omega_{0}$ and the radial wavenumber $\alpha_{0}$. Both of these quantities will be complex-valued in general. In the homogeneous flow case, where $\mu_{1}=0$, the imaginary part of $\omega_{0}$ gives the temporal growth rate that determines whether or not there is absolute instability.

When the flow configuration is taken to be inhomogeneous, it may be seen from (4.2) that the long-term growth behaviour of the impulse solution is dictated by the sign of the quantity

$$
D=\operatorname{Re}\left(\mu_{1}^{2} \gamma\right)
$$

The global stability behaviour thus depends on a balance between the effects of detuning $\operatorname{Im}\left(\mu_{1}\right)$, growth rate variation $\operatorname{Re}\left(\mu_{1}\right)$, diffusion $\operatorname{Re}(\gamma)$ and dispersion $\operatorname{Im}(\gamma)$. If $D$ is positive then the response to the impulse will grow and there will be a global form of instability. However, if $D$ is negative, which is a possibility only when there is detuning so that $\operatorname{Im}\left(\mu_{1}\right) \neq 0$, then there is global stability; for large enough times, the disturbance will decay. This can happen even if the flow is absolutely unstable.

It should be emphasized that our analysis is for linearized disturbances. For the evolution within a realistic flow configuration, nonlinear effects may need to be taken into account. From the analytic form of the impulse solution (4.2), it may easily be surmised that if the magnitude of $D$ is sufficiently small and the initial size of the disturbance happens to be large enough, then temporal amplification associated with absolute stability, which is determined to first order by the growth rate $\operatorname{Im}\left(\omega_{0}\right)$, may suffice to switch on nonlinear effects in the flow; there may not be enough time for any decay that is associated with a negative value of $D$ to come into play.

As in $\S 3$, we can define an instantaneous complex frequency by setting

$$
\omega=\mathrm{i} \frac{1}{G} \frac{\partial G}{\partial t} .
$$

Using (4.2) it may be seen that

$$
\omega=\omega_{0}+\mathrm{i}\left(-\frac{1}{2 t}+\frac{r^{2}}{4 \gamma t^{2}}+\frac{1}{2} \mu_{1} r+\frac{1}{4} \mu_{1}^{2} \gamma t^{2}\right) .
$$

A relationship with this general form can be used to provide a detailed account of the variations in the growth rates and frequencies that were displayed in figure 2. The three complex parameters $\omega_{0}, \gamma$ and $\mu_{1}$ can be chosen by following the same procedure as was described by Thomas \& Davies (2010) for modelling the corresponding behaviour in the rotating-disc flow with mass transfer.

Here, we will adopt a much more succinct approach for making comparisons between the numerical simulation results and the impulse solution obtained from the Ginzburg-Landau equation. If we apply the expression (4.6) to the radial position where the impulse is applied, and also suppose that this is the critical point for the onset of absolute instability in the homogenized flow, then the following simple expression for the instantaneous growth rate is obtained:

$$
\operatorname{Im}(\omega)=-\frac{1}{2 t}+\frac{1}{4} D t^{2} .
$$

It may be recalled that figure 4 displays growth rate plots that were obtained from numerical simulations for various values of the magnetic field parameter $m$, with each 

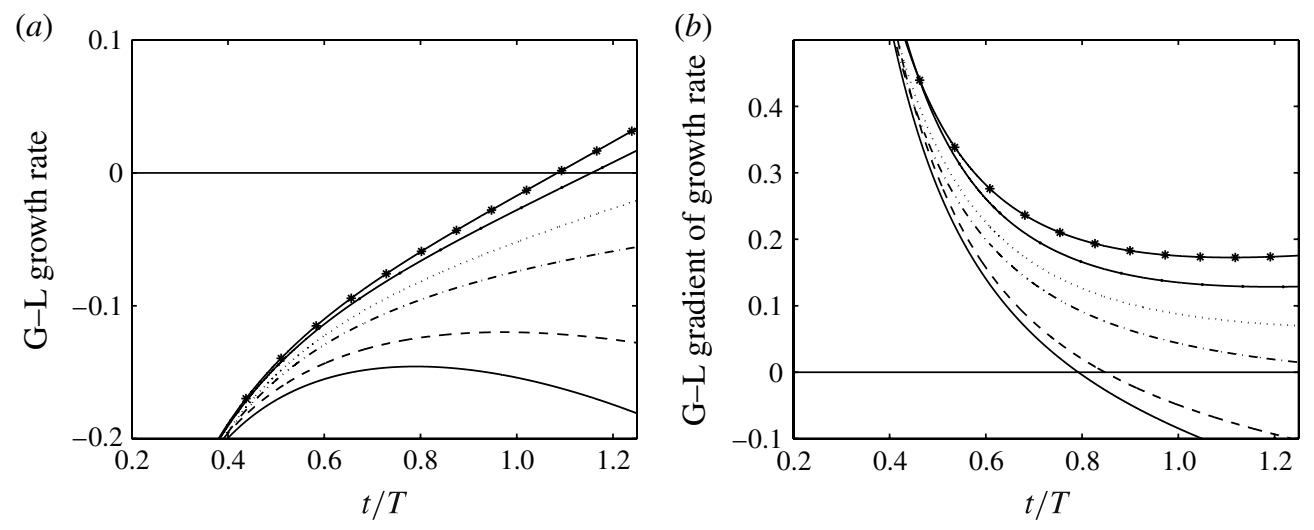

FIGURE 5. Instantaneous growth rates and their gradients for disturbances at the critical point of absolute instability, as given by (4.7) for various values of the parameter $D$. The values of $D$ are chosen so as to fit the simulation data shown in figure 4 . The line types used for different $m$ are preserved between the simulation results and the fitted curves.

of them showing the development at the centre of an impulse that had been applied at the critical point. Thus, if the behaviour discovered in the numerical simulations is well described by the analytic impulse solution that we have been considering, then we should be able to use (4.7) to obtain a good match to each of the curves that are displayed in figure 4 . This should be achievable by making appropriate choices for just one single real parameter $D$, which can be expected to be different for every value of $m$. Moreover, a change in the sign of $D$ from negative to positive, as $m$ is varied, will indicate the switchover from global stability to global instability.

Figure 5 shows plots of growth rates, as well as their gradients, that were determined using (4.7) for various values of the parameter $D$. Although the values of $D$ were chosen to reproduce the variation with $m$ that is shown in figure 4 , no systematic attempt was made to optimize the fit. It can be seen that there is excellent qualitative agreement, as well as a very good degree of quantitative agreement, between the behaviour predicted from the model and that which was discovered in the simulations. It is likely that the small discrepancies that do occur can be traced back to the fact that the impulse applied at the disc surface in the simulations had to be slightly smeared along the radial direction and in time; this is so as to avoid incurring too much computational expense through the use of an unnecessarily high level of numerical resolution.

In agreement with our previous discussion and interpretation of the simulation data in $\S 4$, a change in the sign of $D$ was found when the growth rate curves were fitted for increasing values of $m$, thus indicating the onset of a global instability. By interpolating between the discretely sampled set of $m$ values, it was possible to determine that this onset occurred near to $m=0.2$.

\section{Final remarks}

Numerical simulations of the rotating-disc boundary layer with an imposed axial magnetic field have been carried out. The results of these simulations demonstrate the onset of a globally unstable form of disturbance behaviour. We have investigated the nature of this onset, thus extending the work completed in two recent studies by 
Davies \& Carpenter (2003) and Thomas \& Davies (2010), which were concerned with the non-conducting flow and the effects of mass transfer at the disc surface.

Whilst previous studies, carried out using an homogenized version of the rotatingdisc boundary layer flow, had shown that axial magnetic fields can give rise to a local stabilization, the current investigation indicates that the radial inhomogeneity of the genuine flow can promote globally unstable behaviour when the magnetic field strength is sufficiently large. The contrast between the effects of the magnetic field on the local and global stability behaviour is similar to that which was uncovered by Thomas \& Davies (2010), when they found that locally stabilizing uniform suction could also be a source of global instability.

For disturbances developing in rotating-disc flows modified by mass transfer at the disc surface, it proved possible to cast some light on numerical simulation results by modelling them using simple analytic solutions of a spatially inhomogeneous version of the linearized Ginzburg-Landau equation (Thomas \& Davies 2010). It was concluded that the global disturbance response, following on from an impulsive excitation, was determined by a competition between the effects of frequency detuning, local temporal growth variation and diffusion/dispersion. The same modelling procedure has been applied to the current problem. It was again found that frequency detuning effects played a central role in the explanation of the disturbance behaviour that was obtained in the numerical simulations. In particular, the apparent transition between globally stable and globally unstable responses, as the magnetic field parameter is increased, could be captured by considering the corresponding variation of a single modelling parameter that incorporated the detuning. We surmise that this provides evidence that both the form of the globally unstable behaviour and the nature of its onset are generic.

\section{Acknowledgement}

This work was supported by the Engineering and Physical Sciences Research Council.

\section{REFERENCES}

DAvies, C. \& CARpenter, P. W. 2001 A novel velocity-vorticity formulation of the Navier-Stokes equations with applications to boundary layer disturbance evolution. J. Comput. Phys. 172, $119-165$.

DAvies, C. \& CARPenter, P. W. 2003 Global behaviour corresponding to the absolute instability of the rotating-disc boundary layer. J. Fluid Mech. 486, 287-329.

Davies, C., Thomas, C. \& CARpenter, P. W. 2007 Global stability of the rotating disc boundary layer. J. Engng Maths 57, 219-236.

FALLER, A. J. \& KAYLOR, R. E. 1966 A numerical study of the instability of the laminar Ekman boundary-layer. J. Atmos. Sci. 23, 466-480.

GRAY, W. E. 1952 The nature of the boundary layer at the nose of a swept back wing. Unpublished, Min Aviation, London.

Gregory, N., Stuart, J. T. \& Walker, W. S. 1955 On the stability of three-dimensional boundary-layers with application to the flow due to a rotating disk. Phil. Trans. R. Soc. Lond. Ser. A 248, 155-199.

HeAley, J. J. 2010 Model for unstable global modes in the rotating-disk boundary layer. J. Fluid Mech. 663, 148-159.

HiCKS, T. W. \& RiLEY, N. 1989 Boundary layers in magnetic Czochralski crystal growth. J. Cryst. Growth 96, 957-968.

Hunt, R. E. \& Crighton, D. G. 1991 Instability of flows in spatially developing media. Proc. $R$. Soc. Lond. A 435, 109-128. 
Imayama, S., Alfredsson, P. H. \& Lingwood, R. J. 2013 An experimental study of edge effects on rotating-disk transition. J. Fluid Mech. 716, 638-657.

JASMine, H. A. \& GAJJAR, J. S. B. 2005 Convective and absolute instability in the incompressible boundary layer on a rotating disk in the presence of a uniform magnetic field. J. Engng Maths 52, 337-353.

VON KÁRMÁn, T. 1921 Über laminare und turbulente Reibung. Z. Angew. Math. Mech. 1, 233-252.

KumAR, S. K., THACKeR, W. I. \& WATSON, L. T. 1987 Magnetohydrodynamic flow past a porous rotating disk in a circular magnetic field. Intl J. Numer. Meth. Fluids 8, 659-669.

LINGWOOD, R. J. 1995 Absolute instability of the boundary-layer on a rotating-disk. J. Fluid Mech. 299, 17-33.

LINGWOOD, R. J. 1997 On the effects of suction and injection on the absolute instability of the rotating-disk boundary layers. Phys. Fluids $\mathbf{9}, 1317-1328$.

MACK, L. M. 1985 The wave pattern produced by point source on a rotating disk. AIAA Paper 85-0490.

Organ, A. E. \& Riley, N. 1987 Oxygen transport in magnetic Czochralski growth of silicon. J. Cryst. Growth 82, 465-476.

OthMAn, H. \& CORKE, T. 2006 Experimental investigation of absolute instability of a rotating-disk boundary-layer. J. Fluid Mech. 565, 63-94.

PAO, H. P. 1968 Magnetohydrodynamic flows over a rotating disk. AIAA J. 6, 1285-1291.

SARIC, W. S., ReED, H. L. \& White, E. B. 2003 Stability and transition of three-dimensional boundary layers. Annu. Rev. Fluid Mech. 35, 413-440.

Sparrow, E. M. \& CESS, R. D. 1962 Magnetohydrodynamic and heat transfer about a rotating disk. Trans: ASME J. Appl. Mech. 29, 181-187.

SuryaprakasARAO, U. \& GUPTA, A. S. 1966 Hydromagnetic flow due to a rotating disc subjected to large suction. J. Phys. Soc. Japan 21, 2390-2400.

Thacker, W. I., Watson, L. T. \& Kumar, S. K. 1990 Magnetohydrodynamic free convection from a disc rotating in a vertical plane. Appl. Math. Model. 14, 527-535.

Thomas, C. 2007 Numerical simulations of disturbance development in rotating boundary-layers. PhD thesis, Cardiff University.

Thomas, C., BAssom, A. P. \& Davies, C. 2010 The linear stability of a Stokes layer with an imposed axial magnetic field. J. Fluid Mech. 662, 320-328.

Thomas, C. \& Davies, C. 2010 The effects of mass transfer on the global stability of the rotating-disk boundary layer. J. Fluid Mech. 663, 401-433. 